\title{
RATIONAL HOMOTOPY THEORY: A BRIEF INTRODUCTION
}

\author{
KATHRYN Hess \\ Ecole Polytechnique Fédérale de Lausanne
}

December 2005

\begin{abstract}
These notes contain a brief introduction to rational homotopy theory: its model category foundations, the Sullivan model and interactions with the theory of local commutative rings.
\end{abstract}

\section{INTRODUCTION}

This overview of rational homotopy theory consists of an extended version of lecture notes from a minicourse based primarily on the encyclopedic text [17] of Félix, Halperin and Thomas. With only three hours to devote to such a broad and rich subject, it was difficult to choose among the numerous possible topics to present. Based on the subjects covered in the first week of this summer school, I decided that the goal of this course should be to establish carefully the foundations of the rational homotopy theory, then to treat more superficially one of its most important tools, the Sullivan model. Finally, I provided a brief summary of the extremely fruitful interactions between rational homotopy theory and local algebra, in the spirit of the summer school theme "Interactions between Homotopy Theory and Algebra." I hoped to motivate the students to delve more deeply into the subject themselves, while providing them with a solid enough background to do so with relative ease.

As these lecture notes do not constitute a history of rational homotopy theory, I have chosen to refer the reader to [17], instead of to the original papers, for the proofs of almost all of the results cited, at least in Sections 1 and 2. The reader interested in proper attributions will find them in [17] or [23].

Basic notation and terminology. We assume in this chapter that the reader is familiar with the elements of the theories of simplicial sets and of model categories. As references we recommend [12] and [24] or the chapter of these lecture notes by Paul Goerss [19].

In this chapter, sSet and Top are the categories of simplicial sets and of topological spaces, respectively. Furthermore, $|\cdot|:$ sSet $\longrightarrow$ Top denotes the geometric realization functor, while $S_{\bullet}:$ Top $\longrightarrow$ sSet denotes its right adjoint, the singular simplices functor.

If $K$ is a simplicial set, then $C_{*}(K)$ and $C^{*}(K)$ denote its normalized chain and cochain complexes, respectively. If $X$ is a topological space, then $S_{*}(X):=$ $C_{*}\left(S_{\bullet}(X)\right)$ and $S^{*}(X):=C^{*}\left(S_{\bullet}(X)\right)$, the singular chains and cochains on $X$. 
A morphism of (co)chain complexes inducing an isomorphism in (co)homology is called a quasi-isomorphism and denoted $\stackrel{\sim}{\longrightarrow}$.

A graded vector space is said to be of finite type if it is finite dimensional in each degree.

Given a category $\mathbf{C}$ and two objects $A$ and $B$ in $\mathbf{C}$, we write $\mathbf{C}(A, B)$ for the class of morphisms with source $A$ and target $B$.

\section{Foundations}

For the sake of simplicity, we work throughout these notes only with simply connected spaces. Many of the results presented hold for connected, nilpotent spaces as well.

\subsection{Rationalization and rational homotopy type.}

Definition. A simply connected space $X$ is rational if the following, equivalent conditions are satisfied.

(1) $\pi_{*} X$ is a $\mathbb{Q}$-vector space.

(2) $\mathrm{H}_{*}(X ; \mathbb{Z})$ is a $\mathbb{Q}$-vector space.

$(3) \mathrm{H}_{*}(\Omega X ; \mathbb{Z})$ is a $\mathbb{Q}$-vector space.

Remarks on the proof. To prove the equivalence of these conditions, one begins by observing that $\mathrm{H}_{*}\left(K(\mathbb{Q}, 1) ; \mathbb{F}_{p}\right) \cong \mathrm{H}_{*}\left(\right.$ pt.; $\left.\mathbb{F}_{p}\right)$ for all primes $p$. An inductive Serre spectral sequence argument then shows that $\mathrm{H}_{*}\left(K(\mathbb{Q}, n) ; \mathbb{F}_{p}\right) \cong \mathrm{H}_{*}\left(\mathrm{pt}\right.$.; $\left.\mathbb{F}_{p}\right)$ for all primes $p$ and for all $n \geq 1$. The equivalence of conditions (1) and (2) for an arbitrary $X$ then follows from an inductive argument on the Postnikov tower of $X$.

Example: the rational sphere and disk. For any $n \geq 2$, let $\iota_{n, k}$ denote the homotopy class of the inclusion of $S^{n}$ as the $k^{\text {th }}$ summand of $\bigvee_{k \in \mathbb{N}} S_{k}^{n}$. The rational $n$-sphere is defined to be the complex

$$
S_{0}^{n}:=\left(\bigvee_{k \in \mathbb{N}} S_{k}^{n}\right) \bigcup_{h}\left(\coprod_{l \in \mathbb{N}} D_{l}^{n+1}\right)
$$

where $D_{m}^{n+1}$ is attached to $S_{m-1}^{n} \vee S_{m}^{n}$ by a representative

$$
h_{S_{m}^{n}}: S_{m}^{n} \longrightarrow S_{m-1}^{n} \vee S_{m}^{n}
$$

of $\iota_{n, m}-(m+1) \cdot \iota_{n, m+1}$. The rational $(n+1)$-disk is then

$$
D_{0}^{n+1}:=S_{0}^{n} \times I / S_{0}^{n} \times\{0\} .
$$

Let

$$
X(r)=\left(\bigvee_{1 \leq k \leq r} S_{k}^{n}\right) \bigcup_{h}\left(\coprod_{1 \leq l \leq r-1} D_{l}^{n+1}\right) .
$$

It is clear that for all $r, S_{r}^{n}$ is a strong deformation retract of $X(r)$, which implies that $\mathrm{H}_{k} X(r)=0$ if $k \neq 0, n$. Furthermore, the homomorphism induced in reduced homology by the inclusion $X(r) \hookrightarrow X(r+1)$ is multiplication by $r+1$. Since homology commutes with direct limits and $S_{0}^{n}=\lim _{\rightarrow} X(r)$,

$$
H^{k}\left(S_{0}^{n} ; \mathbb{Z}\right)=\left\{\begin{array}{l}
\mathbb{Z}: k=0 \\
\mathbb{Q}: k=n \\
0: \text { else. }
\end{array}\right.
$$


Definition. A pair of spaces $(X, A)$ is a relative $C W_{0}$-complex if $X=\bigcup_{n \geq 1} X(n)$ where

(1) $X(1)=A$,

(2) for all $n \geq 1$, there is a pushout

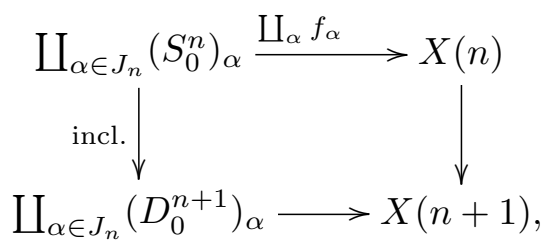

(3) $X$ has the weak topology, i.e., $A \subseteq X$ is open if and only if $A \cap X(n)$ is open in $X(n)$ for all $n$.

The pairs $\left(S_{0}^{n}, S^{n}\right)$ and $\left(D_{0}^{n+1}, D^{n+1}\right)$ are the fundamental examples of relative $C W_{0}$-complexes.

Remark. If $A$ is a rational space and $(X, A)$ is a relative $C W_{0}$-complex, then $X$ is rational as well.

Definition. Let $X$ be a simply connected space. A continuous map $\ell: X \longrightarrow Y$, is a rationalization of $X$ if $Y$ is simply connected and rational and

$$
\pi_{*} \ell \otimes \mathbb{Q}: \pi_{*} X \otimes \mathbb{Q} \longrightarrow \pi_{*} Y \otimes \mathbb{Q} \cong \pi_{*} Y
$$

is an isomorphism.

Remark. A map $\ell: X \longrightarrow Y$ of simply connected spaces is a rationalization if and only if $\mathrm{H}_{*}(\ell ; \mathbb{Q})$ is an isomorphism.

The inclusions of $S^{n}$ into $S_{0}^{n}$ and of $D^{n+1}$ into $D_{0}^{n+1}$ are rationalizations. The rationalization of an abitrary simply connected space, as constructed in the next theorem, generalizes these fundamental examples.

Theorem 1.1.1. Let $X$ be a simply connected space. There exists a relative $C W$ complex $\left(X_{0}, X\right)$ with no zero-cells and no one-cells such that the inclusion $j: X \longrightarrow X_{0}$ is a rationalization. Furthermore, if $Y$ is a simply connected rational space, then any continuous map $f: X \longrightarrow Y$ can be extended over $X_{0}$, i.e., there is a continuous map $g: X_{0} \longrightarrow Y$, which is unique up to homotopy, such that

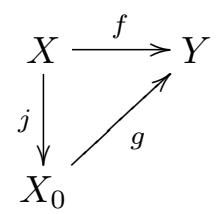

commutes.

Sketch of proof. We can restrict to the case where $X$ is a 1-reduced CW-complex. The rationalization $X_{0}$ can then be constructed as a $\mathrm{CW}_{0}$-complex with rational $n$-cells in bijection with the $n$-cells of $X$, for all $n$. The attaching maps of $X_{0}$ are obtained by rationalizing the attaching maps of $X$. The complete proof can be found in [17], Theorem 9.7. 
Continuing in the same vein, one can show that such a cellular rationalization is unique up to homotopy equivalence relative to $X$.

Given a continuous map $\varphi: X \longrightarrow Y$ between simply connected spaces, we let $\varphi_{0}: X_{0} \longrightarrow Y_{0}$ denote the induced map between their rationalizations, the existence and uniqueness (up to homotopy) of which is guaranteed by the theorem above.

Definition. The rational homotopy type of a simply connected space $X$ is the weak homotopy type of $X_{0}$.

Definition. A continuous map $\varphi: X \longrightarrow Y$ between simply connected spaces is a rational homotopy equivalence if the following, equivalent conditions are satisfied.

(1) $\pi_{*} \varphi \otimes \mathbb{Q}$ is an isomorphism.

(2) $\mathrm{H}_{*}(\varphi ; \mathbb{Q})$ is an isomorphism.

(3) $\mathrm{H}^{*}(\varphi ; \mathbb{Q})$ is an isomorphism.

(4) $\varphi_{0}: X_{0} \longrightarrow Y_{0}$ is a weak homotopy equivalence.

To facilitate computations, it is common in rational homotopy theory to restrict to the class of spaces defined by the following proposition, the proof of which is in [17], Theorem 9.11.

Proposition 1.1.2. For any simply connected space $X$, there is $C W$-complex $Z$ and a rational homotopy equivalence $\varphi: Z \longrightarrow X$ such that

(1) $\mathrm{H}_{*}(X ; \mathbb{Q})$ is of finite type if and only if $Z$ is of finite type; and

(2) if $\operatorname{dim}_{\mathbb{Q}} \mathrm{H}_{*}(X ; \mathbb{Q})<\infty$, then $\mathrm{H}_{*}(X ; \mathbb{Q})=H_{\leq N}(X ; \mathbb{Q})$ if and only if $Z$ is a finite $C W$-complex of dimension at most $N$.

Definition. A simply connected space $X$ is of finite rational type if condition (1) of Proposition 1.1.2 is satisfied.

We can now finally specify clearly the subject presented in these notes.

\section{Rational homotopy theory is the study of rational homotopy types of spaces and of the properties of spaces and maps that are invariant under rational homotopy equivalence.}

For further information on rationalization, the reader is refered to section 9 of [17].

\subsection{The passage to commutative cochain algebras.}

We show in this section that the category of rational homotopy types of simply connected, finite-type spaces and of homotopy classes of maps between their representatives is equivalent to an appropriately defined homotopy category of commutative differential graded algebras over $\mathbb{Q}$.

The algebraic category and its homotopy structure. We begin by a rather careful introduction to the algebraic category in which the Sullivan model of a topological space lives.

A commutative differential graded algebra (CDGA) over $\mathbb{Q}$ is a commutative monoid in the category of non-negatively graded, rational cochain complexes. In other words, a CDGA is a cochain complex $\left(A^{*}, d\right)$ over $\mathbb{Q}$, endowed with cochain maps

$$
\eta: \mathbb{Q} \longrightarrow\left(A^{*}, d\right)
$$


called the unit and

$$
\mu:\left(A^{*}, d\right) \otimes_{\mathbb{Q}}\left(A^{*}, d\right) \longrightarrow\left(A^{*}, d\right): a \otimes b \longmapsto \longrightarrow a \cdot b,
$$

called the product such that

(1) $\mu$ is graded commutative, i.e., if $a \in A^{p}$ and $b \in A^{q}$, then $a \cdot b=(-1)^{p q} b \cdot a$;

(2) $\mu$ is associative; and

(3) $\mu\left(\eta \otimes 1_{A}\right)=1_{A}=\mu\left(1_{A} \otimes \eta\right)$.

Let $r \geq 0$. A CDGA $A$ is $r$-connected if $A^{0}=\mathbb{Q}$ and $A^{k}=0$ for all $0<k<r+1$.

A morphism of CDGA's $f:\left(A^{*}, d, \mu, \eta\right) \longrightarrow\left(\bar{A}^{*}, \bar{d}, \bar{\mu}, \bar{\eta}\right)$ is a cochain map such that $f \mu=\bar{\mu}(f \otimes f)$ and $f \eta=\bar{\eta}$. The category of CDGA's over $\mathbb{Q}$ and their morphisms is denoted CDGA $\mathbf{A}_{\mathbb{Q}}$.

To simplify notation, we frequently write either $A$ or $(A, d)$ to denote $\left(A^{*}, d, \mu, \eta\right)$. Furthermore, henceforth in these notes, the notation $\otimes$ means the tensor product over $\mathbb{Q}$.

In rational homotopy theory, CDGA's with free underlying commutative, graded algebra play an essential role. Given a non-negatively graded vector space $V=$ $\bigoplus_{i \geq 0} V^{i}$, let $\Lambda V$ denote the free, commutative, graded algebra generated by $V$, i.e.,

$$
\Lambda V=S\left[V^{\mathrm{even}}\right] \otimes E\left(V^{\mathrm{odd}}\right),
$$

the tensor product of the symmetric algebra on the vectors of even degree and of the exterior algebra on the vectors of odd degree. Given a basis $\left\{v_{j} \mid j \in J\right\}$ of $V$, we often write $\Lambda\left(v_{j}\right)_{j \in J}$ for $\Lambda V$.

A homomorphism of commutative, graded algebras $\varphi: \Lambda V \longrightarrow A$ is determined by its restriction to $V$, as is any derivation of commutative, graded algebras $\delta: \Lambda V \longrightarrow A$. In particular, the differential $d$ of a CDGA $(\Lambda V, d)$ is determined by its restriction to $V$.

More generally, the following class of CDGA morphisms is particularly important in rational homotopy theory.

Definition. A relative Sullivan algebra consists of an inclusion of CDGA's $(A, d) \hookrightarrow$ $(A \otimes \Lambda V, D)$ such that $V$ has a basis $\left\{v_{\alpha} \mid \alpha \in J\right\}$, where $J$ is a well-ordered set, such that $d v_{\beta} \in A \otimes \Lambda V_{<\beta}$ for all $\beta \in J$, where $V_{<\beta}$ is the span of $\left\{v_{\alpha} \mid \alpha<\beta\right\}$. A relative Sullivan algebra $(A \otimes \Lambda V, D)$ is minimal if

$$
\alpha<\beta \Rightarrow \operatorname{deg} v_{\alpha} \leq \operatorname{deg} v_{\beta} .
$$

A (minimal) relative Sullivan algebra $(\Lambda V, d)$ extending $(A, d)=(\mathbb{Q}, 0)$ is called a (minimal) Sullivan algebra.

Remark. If $V^{0}=0=V^{1}$, then $(\Lambda V, d)$ is minimal if and only if $d V \subseteq \Lambda^{\geq 2} V$.

Example. The CDGA $(\Lambda(x, y, z), d)$, where $x, y$ and $z$ are all of degree 1 and $d x=y z, d y=z x$ and $d z=y x$ is an example of CDGA with free underlying graded algebra that is not a Sullivan algebra.

Recall from Example 1.7 and Example 3.4 (1) in [19] that the category $\mathbf{C h}(\mathbb{Q})$ of non-negatively graded cochain complexes over $\mathbb{Q}$ admits a cofibrantly generated model category structure in which

(1) weak equivalences are quasi-isomorphisms;

(2) fibrations are degreewise surjections; and

(3) cofibrations are degreewise injections, in positive degrees. 
The set of generating acyclic cofibrations is

$$
\mathcal{J}=\left\{j_{n}: 0 \rightarrow D(n) \mid n \geq 1\right\}
$$

where

$$
D(n)_{k}=\left\{\begin{array}{l}
\mathbb{Q}: k=n-1, n \\
0: \text { else }
\end{array}\right.
$$

and $d_{n-1}: D(n)_{n-1} \rightarrow D(n)_{n}$ is the identity map. The set $\mathcal{I}$ of generating cofibrations consists of the obvious inclusions

$$
i_{n}: S(n-1) \rightarrow D(n) \text { for } n \geq 1 \quad \text { and } \quad i_{0}: 0 \rightarrow S(0)
$$

where

$$
S(n-1)_{k}=\left\{\begin{array}{l}
\mathbb{Q}: k=n-1 \\
0: \text { else }
\end{array}\right.
$$

Consider the pair of adjoint functors

$$
\Lambda: \mathbf{C h}^{*}(\mathbb{Q}) \rightleftarrows \mathbf{C D G A}_{\mathbb{Q}}: U,
$$

where $\Lambda$ is the "free commutative cochain algebra functor" satisfying $\Lambda(C, d)=$ $(\Lambda C, \tilde{d})$, where $\tilde{d}$ is the derivation extending $d$, and $U$ is the forgetful functor. It is not difficult, as indicated in Example 3.7 in [19], to show that this adjoint pair satisfies the hypotheses of Theorem 3.6 of [19].

There is thus a cofibrantly generated model structure on CDGA $\mathbf{A}_{\mathbb{Q}}$, with generating cofibrations and acyclic cofibrations

$$
\mathcal{I}=\left\{\Lambda i_{n} \mid n \geq 0\right\} \text { and } \mathcal{J}=\left\{\Lambda j_{n} \mid n \geq 1\right\}
$$

where $\Lambda(0):=\mathbb{Q}$. Let $\mathcal{I}-$ cell denote the smallest class of morphisms in CDGA $\mathbf{A}_{\mathbb{Q}}$ that contains $\mathcal{I}$ and that is closed under coproducts, cobase change and sequential colimits. It is easy to see that $\mathcal{I}$-cell is exactly the class of relative Sullivan algebras. In this model structure on $\mathbf{C D G} \mathbf{A}_{\mathbb{Q}}$, weak equivalences are quasi-isomorphisms, and fibrations are degreewise surjections. Cofibrations are retracts of relative Sullivan algebras. All CDGA's are fibrant, and the Sullivan algebras are the cofibrant CDGA's.

The next result, which rephrases the Lifting Axiom of model categories in the specific case of $\mathbf{C D G} \mathbf{A}_{\mathbb{Q}}$, is an important tool in rational homotopy theory.

Proposition 1.2.1 (The Lifting Lemma). Let

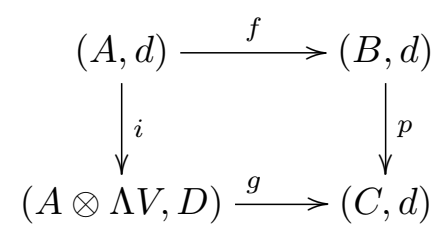

be a commuting diagram in $\mathbf{C D G} \mathbf{A}_{\mathbb{Q}}$, where $i$ is a relative Sullivan algebra and $p$ is a surjection. If $i$ or $p$ is a quasi-isomorphism, then $g$ lifts through $p$ to an extension of $f$, i.e., there exists a CDGA map $h:(A \otimes \Lambda V, D) \rightarrow(B, d)$ such that $h i=f$ and $p h=g$. Furthermore, any two lifts are homotopic rel $A$.

We can describe homotopy of CDGA morphisms with source a Sullivan algebra in terms of the following path objects. Let $I$ denote the CDGA $(\Lambda(t, y), d)$, where $\operatorname{deg} t=0, \operatorname{deg} y=1$ and $d t=y$. Let $\varepsilon_{0}: I \rightarrow \mathbb{Q}$ and $\varepsilon_{1}: I \rightarrow \mathbb{Q}$ denote the augmentations specified by $\varepsilon_{0}(t)=0$ and $\varepsilon_{1}(t)=1$. 
Proposition 1.2.2. Let $(\Lambda V, d)$ be a Sullivan algebra. Two CDGA morphisms $f, g:(\Lambda V, d) \rightarrow(A, d)$ are homotopic if and only if there is a CDGA morphism $H:(\Lambda V, d) \rightarrow(A, d) \otimes I$ such that $\left(I d \otimes \varepsilon_{0}\right) H=f$ and $\left(I d \otimes \varepsilon_{1}\right) H=g$.

A careful, degree-by-degree version of the proof of the Small Object Argument (Theorem 3.5 in [19]) establishes the following useful result.

Proposition 1.2.3. Any morphism $f:(A, d) \rightarrow(B, d)$ in CDGA $_{\mathbb{Q}}$ can be factored as

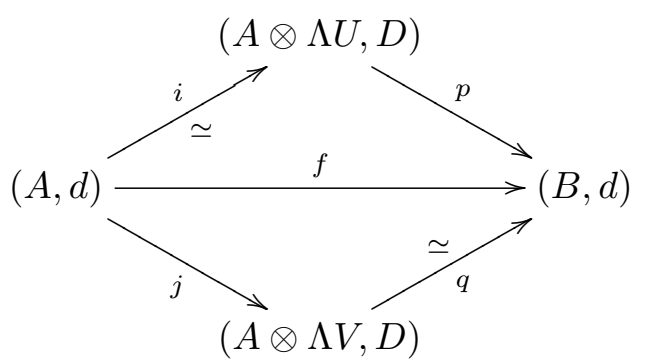

where $i$ and $j$ are relative Sullivan algebras, and $p$ and $q$ are surjections.

If we are willing to sacrifice surjectivity of $q$, we can obtain minimality of $j$, again via a degree-by-degree construction.

Proposition 1.2.4. Any morphism $f:(A, d) \rightarrow(B, d)$ in CDGA $_{\mathbb{Q}}$ can be factored as

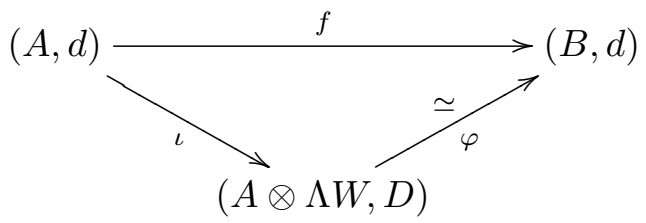

where $\iota$ is a minimal relative Sullivan algebra. In particular, if $H^{0} A=\mathbb{Q}=H^{0} B$, $H^{1} f$ is injective, and $H^{*} B$ is of finite type, then $W$ is of finite type and $W=W \geq 2$.

Definition. The quasi-isomorphism $\varphi:(A \otimes \Lambda W, D) \stackrel{\simeq}{\longrightarrow}(B, d)$ is a relative Sullivan minimal model of $f: A \rightarrow B$. A Sullivan minimal model of the CDGA $(B, d)$ is a relative Sullivan minimal model $\varphi:(\Lambda W, d) \stackrel{\simeq}{\rightarrow}(B, d)$ of the unit map $\eta:(\mathbb{Q}, 0) \rightarrow(B, d)$.

It is very convenient to know that (relative) Sullivan minimal models are unique up to isomorphism, which is an immediate consequence of the following proposition.

Proposition 1.2.5. Suppose that

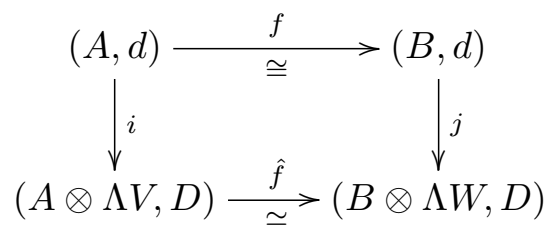

is a commuting diagram in $\mathbf{C D G A}_{\mathbb{Q}}$, where $i$ and $j$ are minimal relative Sullivan algebras, $f$ is an isomorphism and $\hat{f}$ is a quasi-isomorphism. Then $\hat{f}$ is also an isomorphism. 
The proof of this proposition reduces to showing that if a CDGA endomorphism of a minimal relative Sullivan algebra $(B \otimes \Lambda W, D)$ fixing $B$ is homotopic to the identity, then it is equal to the identity.

The functors. We now explain the passage from topology to algebra, starting with the relationship between simplicial sets and CDGA's.

Definition. The algebra of polynomial differential forms, $\mathfrak{A}_{\bullet}^{*}$, is the simplicial CDGA given by

$$
\mathfrak{A}_{n}^{*}=\left(\Lambda\left(t_{0}, \ldots, t_{n} ; y_{0}, \ldots, y_{n}\right) / J_{n}, d\right)
$$

where $\operatorname{deg} t_{i}=0$ and $d t_{i}=y_{i}$ for all $i$ and $J_{n}$ is the ideal generated by $\{1-$ $\left.\sum_{i=0}^{n} t_{i}, \sum_{j=0}^{n} y_{j}\right\}$, and the faces and degeneracies are specified by

$$
\partial_{i}: \mathfrak{A}_{n}^{*} \longrightarrow \mathfrak{A}_{n-1}^{*}: t_{k} \longmapsto\left\{\begin{array}{l}
t_{k}: k<i \\
0 \quad: k=i \\
t_{k-1}: k>i
\end{array}\right.
$$

and

$$
s_{i}: \mathfrak{A}_{n}^{*} \longrightarrow \mathfrak{A}_{n+1}^{*}: t_{k} \longmapsto \begin{cases}t_{k} & : k<i \\ t_{k}+t_{k+1}: k=i \\ t_{k+1} & : k>i .\end{cases}
$$

The terminology used in this definition is justified by the following observation. Let $\Omega_{\mathrm{DR}}\left(\Delta^{n}\right)$ be the cochain algebra of smooth forms on $\Delta^{n}$, the standard topological $n$-simplex. Then

$$
\Omega_{\mathrm{DR}}\left(\Delta^{n}\right)=C^{\infty}\left(\Delta^{n}\right) \underset{\mathfrak{A}_{n}^{0}}{\otimes} \mathfrak{A}_{n}^{*},
$$

where the induced face and degeneracy maps satisfy $\partial_{i}=\Omega_{\mathrm{DR}}\left(i^{\text {th }}\right.$ face inclusion $)$ and $s_{i}=\Omega_{\mathrm{DR}}\left(i^{\text {th }}\right.$ degeneracy $)$.

Definition. Let $\mathcal{A}^{*}$ : sSet $\longrightarrow$ CDGA be the functor specified by $\mathcal{A}^{*}(K)=$ $\operatorname{sSet}\left(K, \mathfrak{A}_{\bullet}^{*}\right)$, with operations defined objectwise. For any topological space, let $\mathcal{A}_{P L}(X):=\mathcal{A}^{*}\left(S_{\bullet}(X)\right)$, which we call the CDGA of piecewise-linear de Rham forms on $X$.

Since for any simplicial set $K, \mathcal{A}^{*}(K)$ is a commutative algebra, while $C^{*}(K ; \mathbb{Q})$ is usually not, we cannot expect to be able to define a natural quasi-isomorphism of cochain algebras directly from the former to the latter. However, as explained below, there is a cochain map between them that is close to being an algebra map.

Given $f \in \mathcal{A}^{n}(K)$, i.e., $f: K \longrightarrow \mathfrak{A}_{\bullet}^{n}$, and $x \in K_{n}$, write

$$
f(x)=\hat{f}(x) d t_{1} \cdots d t_{n},
$$

so that $\hat{f}(x) \in \mathbb{Q}\left[t_{1}, \ldots, t_{n}\right]$. Define a graded linear map $\oint: \mathcal{A}^{*}(K) \longrightarrow C^{*}(K ; \mathbb{Q})$ by

$$
(\oint f)(x)=\int_{\Delta^{n}} \hat{f}(x) d t_{1} \cdots d t_{n} .
$$


Theorem 1.2.6 (Polynomial Stokes-DeRham Theorem). The map $\oint$ is a map of cochain complexes, inducing an isomorphism of algebras in cohomology.

The proof of this theorem, which can be found in [9], Theorem 2.2 and Corollary 3.4 , proceeds by methods of acyclic models.

Theorem 1.2.6 can in fact be strengthened: as proved in Proposition 3.3 in [9], the cochain map $\oint$ is actually a strongly homotopy multiplicative map, in the sense of e.g., Gugenheim and Munkholm [20].

To compare the homotopy theory of CDGA's and of simplicial sets via the functor $\mathcal{A}^{*}$, we need for $\mathcal{A}^{*}$ to be a member of an adjoint pair. We construct its adjoint as follows.

Definition. Let $\mathcal{K}_{\bullet}:$ CDGA $\longrightarrow$ sSet be the functor specified by $\mathcal{K}_{\bullet}(A)=$ $\operatorname{CDGA}\left(A, \mathfrak{A}_{\bullet}^{*}\right)$, with faces and degeneracies defined objectwise.

Let sCDGA denote the category of simplicial CDGA's. Given any CDGA $A$ and any simplicial set $K$, their cartesian product $A \times K$ is naturally a simplicial CDGA. Furthermore, there are natural isomorphisms

$$
\operatorname{CDGA}\left(A, \operatorname{sSet}\left(K, \mathfrak{A}_{\bullet}^{*}\right)\right) \cong \operatorname{sCDGA}\left(A \times K, \mathfrak{A}_{\bullet}^{*}\right) \cong \operatorname{sSet}\left(K, \operatorname{CDGA}\left(A, \mathfrak{A}_{\bullet}^{*}\right)\right),
$$

i.e.,

$$
\operatorname{CDGA}\left(A, \mathcal{A}^{*}(K)\right) \cong \operatorname{sSet}\left(K, \mathcal{K}_{\bullet}(A)\right) .
$$

We therefore have an adjoint pair

$$
\mathcal{A}^{*}: \text { sSet } \rightleftarrows \text { CDGA }^{o p}: \mathcal{K}_{\bullet},
$$

which Bousfield and Gugenheim proved to be a Quillen pair in Section 8 of [9].

Definition. The composite functor

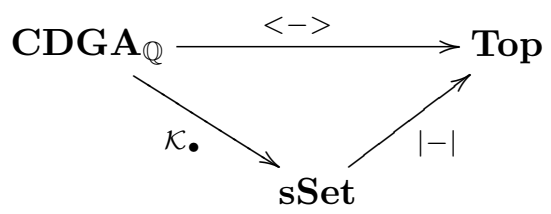

is called spatial realization.

Let $(\Lambda V, d)$ be any Sullivan algebra. Consider the commuting diagram

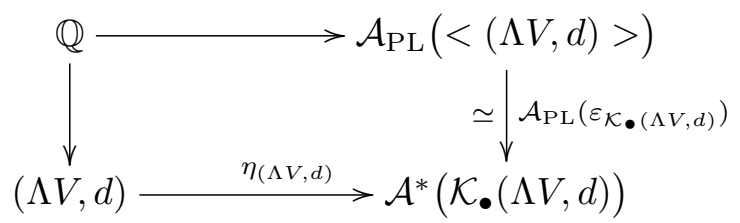

where $\eta: I d \rightarrow \mathcal{A}^{*} \circ \mathcal{K}_{\bullet}$ is the unit of the adjoint pair (1.2.1) and $\varepsilon: S_{\bullet} \circ \mid-$ $\mid \rightarrow I d$ is the counit of the adjoint pair $\left(S_{\bullet},|-|\right)$. Since $(\Lambda V, d)$ is a Sullivan algebra and $\mathcal{A}_{\mathrm{PL}}\left(\varepsilon_{\mathcal{K}_{\bullet}(\Lambda V, d)}\right)$ is a surjective quasi-isomorphism, the Lifting Lemma (Proposition 1.2.1) can be applied to this diagram, establishing the existence of a CDGA morphism $m_{(\Lambda V, d)}:(\Lambda V, d) \rightarrow \mathcal{A}_{\mathrm{PL}}(<(\Lambda V, d)>)$, unique up to homotopy, lifting $\eta_{(\Lambda V, d)}$. 
Theorem 1.2.7. If $(\Lambda V, d)$ is a simply connected Sullivan algebra of finite type, then

(1) $m_{(\Lambda V, d)}:(\Lambda V, d) \rightarrow \mathcal{A}_{\mathrm{PL}}(<(\Lambda V, d)>)$ is a quasi-isomorphism; and

(2) $<(\Lambda V, d)>$ is a simply connected, rational space of finite type, such that there is an isomorphism of graded rational vector spaces

$$
\pi_{*}(<(\Lambda V, d)>) \cong \operatorname{Hom}_{\mathbb{Q}}(V, \mathbb{Q}) .
$$

We refer the reader to Section 17 of [17] for the details of the proof of this extremely important theorem.

To complete the picture, we need to specify the relationship between spatial realization and homotopy of morphisms.

Theorem 1.2.8. Let $(\Lambda V, d)$ and $(\Lambda W, d)$ be simply connected Sullivan algebras of finite type.

(1) Let $f:(\Lambda V, d) \rightarrow(\Lambda W, d)$ be a CDGA morphism. Then

$$
\begin{aligned}
& (\Lambda V, d) \longrightarrow(\Lambda W, d) \\
& \simeq m_{(\Lambda V, d)} \quad \quad \quad \quad \operatorname{AP}_{\mathrm{PL}}(<f>) \quad m_{(\Lambda W, d)} \\
& \mathcal{A}_{\mathrm{PL}}(<(\Lambda V, d)>) \stackrel{\mathcal{A}_{\mathrm{PL}}(<f>)}{\longrightarrow} \mathcal{A}_{\mathrm{PL}}(<(\Lambda W, d)>)
\end{aligned}
$$

commutes up to homotopy.

(2) Two CDGA morphisms $f, g:(\Lambda V, d) \rightarrow(\Lambda W, d)$ are homotopic if and only if $\langle f\rangle$ and $\langle g\rangle$ are homotopic.

(3) Let $\alpha: X \rightarrow Y$ be a continuous map between simply connected $C W$ complexes of finite rational type. If there is a homotopy-commutative diagram of $C D G A$ 's

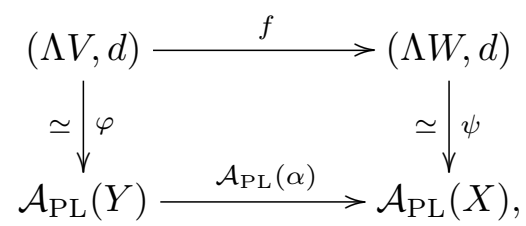

then there is a homotopy-commutative diagram

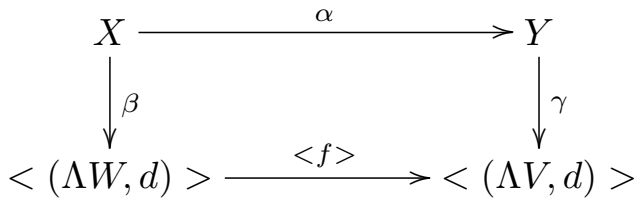

in which $\pi_{*}(\beta) \otimes \mathbb{Q}$ and $\pi_{*}(\gamma) \otimes \mathbb{Q}$ are isomorphims.

Again, we refer the reader to Section 17 in [17] for the proof of this theorem. 
Corollary 1.2.9. Rational homotopy types of simply connected spaces of finite rational type are in bijective correspondence with isomorphism classes of minimal Sullivan algebras. Furthermore, homotopy classes of continuous maps of simply connected, finite-type rational spaces are in bijective correspondence with homotopy classes of morphisms of simply connected, finite-type Sullivan algebras.

We are now ready to introduce one of the very most important tools in rational homotopy theory.

Definition. The Sullivan minimal model of a simply connected topological space $X$ of finite rational type is the unique (up to isomorphism) Sullivan minimal model of its algebra of piecewise-linear de Rham forms

$$
\varphi:(\Lambda V, d) \stackrel{\simeq}{\longrightarrow} \mathcal{A}_{\mathrm{PL}}(X) .
$$

As a consequence of Theorems 1.2.7 and 1.2.8, if $\varphi:(\Lambda V, d) \stackrel{\simeq}{\longrightarrow} \mathcal{A}_{\mathrm{PL}}(X)$ is a Sullivan minimal model, then there is isomorphism of graded rational vector spaces

$$
\operatorname{Hom}_{\mathbb{Q}}(V, \mathbb{Q}) \cong \pi_{*} X \otimes \mathbb{Q} .
$$

In other words, given a Sullivan minimal model of a space, we can read off the nontorsion part of its homotopy groups from the generators of the model.

\section{Sullivan MODElS}

Since the CDGA $\mathcal{A}_{\mathrm{PL}}(X)$ is huge and has a complicated product, rational homotopy theorists prefer to carry out computations with the Sullivan minimal model, which has only finitely many generators in each dimension if $X$ is of finite rational type and which is free as an algebra. In this section, we provide a brief overview of the power of the Sullivan model. We begin by providing a few explicit examples of Sullivan minimal models. We then explore the relationship between topological fibrations and the Sullivan model. In particular, we explain the slogan "the Sullivan model of fiber is the cofiber of the Sullivan model" and illustrate its application. A classical and essential numerical homotopy invariant, Lusternik-Schnirelmann category, is our next subject: its elementary properties, how to calculate it using the Sullivan model and its additivity. Finally, we present the beautiful and striking rational dichotomy of finite $\mathrm{CW}$-complexes, the proof of which depends crucially on Lusternik-Schnirelmann category.

\subsection{Examples and elementary constructions.}

As a warmup and an aid to developing the reader's intuition, we calculate a few explicit examples of Sullivan models. Here, a subscript on a generator always indicates its degree.

Spheres. The Sullivan model of an odd sphere $S^{2 n+1}$ is

$$
\varphi:\left(\Lambda\left(x_{2 n+1}\right), 0\right) \rightarrow \mathcal{A}_{\mathrm{PL}}\left(S^{2 n+1}\right),
$$

where $\varphi(x)$ is any representative of the unique cohomology generator of degree $2 n+1$. Since $\varphi$ is obviously a quasi-isomorphism of CDGA's, the nontorsion part of $\pi_{*} S^{2 n+1}$ is concentrated in degree $2 n+1$, where it is of rank 1 . 
On the other hand, the Sullivan model of an even sphere $S^{2 n}$ is

$$
\varphi:\left(\Lambda\left(y_{2 n}, z_{4 n-1}\right), d\right) \rightarrow \mathcal{A}_{\mathrm{PL}}\left(S^{2 n}\right)
$$

where $d z=y^{2}$ and $\varphi(y)$ represents the unique cohomology generator of degree $2 n$. Since the square of $\varphi(y)$ must be a boundary, there is an acceptable choice of $\varphi(z)$. Again, $\varphi$ is clearly a quasi-isomorphism of CDGA's, implying that the nontorsion part of $\pi_{*} S^{2 n}$ is concentrated in degrees $2 n$ and $4 n-1$ and that it is of rank 1 in each of those degrees.

Complex projective spaces. From the long exact sequences in homotopy of the fibrations

$$
S^{1} \rightarrow S^{2 n+1} \rightarrow \mathbb{C} P^{n}
$$

and

$$
S^{1} \rightarrow S^{\infty} \rightarrow \mathbb{C} P^{\infty}
$$

and the computation above of $\pi_{*} S^{2 n+1} \otimes \mathbb{Q}$, we conclude that

$$
\pi_{*} \mathbb{C} P^{n} \otimes \mathbb{Q}=\mathbb{Q} \cdot u_{2} \oplus \mathbb{Q} \cdot x_{2 n+1} \text { and } \pi_{*} \mathbb{C} P^{\infty} \otimes \mathbb{Q}=\mathbb{Q} \cdot u_{2} .
$$

Consequently, the Sullivan model of $\mathbb{C} P^{n}$ is of the form

$$
\varphi:\left(\Lambda\left(u_{2}, x_{2 n+1}\right), d\right) \rightarrow \mathcal{A}_{\mathrm{PL}}\left(\mathbb{C} P^{n}\right)
$$

where $d x=u^{n+1}, \varphi(u)$ represents the algebra generator of $H^{*}\left(\mathbb{C} P^{n} ; \mathbb{Q}\right)$, which is a truncated polynomial algebra on a generator of degree 2 , and $\varphi(x)$ kills its $(n+1)^{s t}$ power. The value of $d x$ is nonzero since $H^{*}\left(\mathbb{C} P^{n} ; \mathbb{Q}\right)$ is zero in odd degrees.

The Sullivan model for $\mathbb{C} P^{\infty}$ is even easier to specify since there can be no nontrivial differential. It is

$$
\varphi:(\Lambda(u), 0) \rightarrow \mathcal{A}_{\mathrm{PL}}\left(\mathbb{C} P^{\infty}\right)
$$

where $\varphi(u)$ represents the algebra generator of $H^{*}\left(\mathbb{C} P^{\infty} ; \mathbb{Q}\right)$, which is a polynomial algebra on a generator of degree 2

Products. Observe that $\mathcal{A}_{\mathrm{PL}}$ is a lax monoidal functor, via the natural quasiisomorphism $\alpha_{X, Y}$, defined to be the composite

$\mathcal{A}_{\mathrm{PL}}(X) \otimes \mathcal{A}_{\mathrm{PL}}(Y) \stackrel{\mathcal{A}_{\mathrm{PL}}\left(p_{1}\right) \otimes \mathcal{A}_{\mathrm{PL}}\left(p_{2}\right)}{\longrightarrow} \mathcal{A}_{\mathrm{PL}}(X \times Y) \otimes \mathcal{A}_{\mathrm{PL}}(X \times Y) \stackrel{\mu}{\longrightarrow} \mathcal{A}_{\mathrm{PL}}(X \times Y)$,

where $p_{i}$ is projection onto the $i^{\text {th }}$ component, and $\mu$ is the product on $\mathcal{A}_{\mathrm{PL}}(X \times Y)$.

Given Sullivan models $\varphi:(\Lambda V, d) \rightarrow \mathcal{A}_{\mathrm{PL}}(X)$ and $\varphi^{\prime}:\left(\Lambda V^{\prime}, d^{\prime}\right) \rightarrow \mathcal{A}_{\mathrm{PL}}\left(X^{\prime}\right)$, the Sullivan model of the product space $X \times X^{\prime}$ is given by

$$
(\Lambda V, d) \otimes\left(\Lambda V^{\prime}, d^{\prime}\right) \stackrel{\varphi \otimes \varphi^{\prime}}{\longrightarrow} \mathcal{A}_{\mathrm{PL}}(X) \otimes \mathcal{A}_{\mathrm{PL}}\left(X^{\prime}\right) \stackrel{\alpha_{X, X^{\prime}}}{\longrightarrow} \mathcal{A}_{\mathrm{PL}}\left(X \times X^{\prime}\right) .
$$

Formal spaces. A space is formal if its rational homotopy is a formal consequence of its rational cohomology, in the sense of the following definition. 
Definition. A rational CDGA $A$ is formal if there is a quasi-isomorphism of cochain algebras $A \longrightarrow \mathrm{H}^{*}(A)$. A space $X$ is formal if $\mathcal{A}_{\mathrm{PL}}(X)$ is a formal CDGA.

From the previous examples, we see that spheres and complex projective spaces are formal. Furthermore, products of formal spaces are clearly formal. We can also show that wedges of formal spaces are formal, as follows.

Observe that for any family of pointed spaces $\left\{X_{j} \mid j \in J\right\}$,

$$
\begin{aligned}
\mathcal{A}_{\mathrm{PL}}\left(\bigvee_{j \in J} X_{j}\right) & =\mathcal{A}^{*}\left(S_{\bullet}\left(\bigvee_{j \in J} X_{j}\right)\right)=\operatorname{sSet}\left(S_{\bullet}\left(\bigvee_{j \in J} X_{j}\right), \mathfrak{A}_{\bullet}^{*}\right) \\
& =\operatorname{sSet}\left(\bigvee_{j \in J} S_{\bullet}\left(X_{j}\right), \mathfrak{A}_{\bullet}^{*}\right)=\prod_{j \in J} \operatorname{sSet}\left(S_{\bullet}\left(X_{j}\right), \mathfrak{A}_{\bullet}^{*}\right)=\prod_{j \in J} \mathcal{A}_{\mathrm{PL}}\left(X_{j}\right) .
\end{aligned}
$$

Since a product of formal CDGA's is clearly formal, we obtain that a wedge of formal spaces is formal, too.

Further examples of formal spaces can be found in geometry. Given a compact, connected Lie group $G$, let $K$ denote the connected component of its neutral element $e$, in the subgroup of elements fixed by a given involution. The quotient $G / K$, which is a symmetric space, is then a formal space, as proved in [10]. Furthermore, Deligne, Griffiths, Morgan and Sullivan showed in [11] that compact Kähler manifolds are also formal.

It is easy to construct an example of a nonformal CDGA. Let $A=(\Lambda(u, v, w), d)$, where $|u|=|v|=3$ and $|w|=5$ and where $d w=u v$. Then

$$
\mathrm{H}_{n}(A)= \begin{cases}\mathbb{Q} & : n=0,11 \\ \mathbb{Q} \oplus \mathbb{Q}: n=3,8 & : \text { else },\end{cases}
$$

where the classes in degree 3 are represented by $u w$ and $v w$ and the class in degree 11 by uvw. If $\varphi: A \longrightarrow \mathrm{H}_{*}(A)$ is a CDGA map, then $\varphi(w)=0$ for degree reasons, which implies that $\varphi(u w)=0=\varphi(v w)$, since $\varphi$ is an algebra map. Consequently, $\varphi$ cannot be a quasi-isomorphism.

\subsection{Models of fiber squares.}

The Sullivan model is especially well adapted to studying fibrations. In particular, as expressed more precisely in the next theorem, "the Sullivan model of a fiber is the cofiber of the model."

Theorem 2.2.1. Let $p: E \longrightarrow B$ be a Serre fibration such that $B$ is simply connected and $E$ is path connected. Let $F$ denote the fiber of $p$. Suppose that B or F is of finite rational type.

(1) Given a Sullivan model $\mu:(\Lambda V, d) \longrightarrow \mathcal{A}_{\mathrm{PL}}(B)$, let

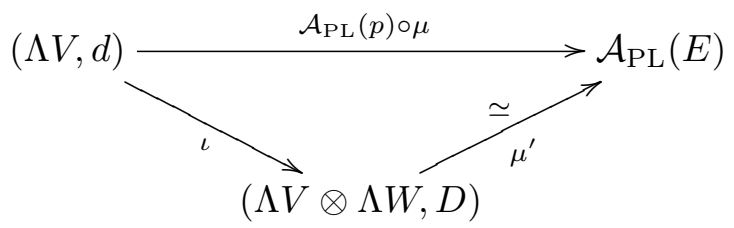

be a factorization of $\mathcal{A}_{\mathrm{PL}}(p) \circ \mu$ as a relative Sullivan algebra, followed by a quasi-isomorphism. Let $(\Lambda W, \bar{D})=\mathbb{Q} \otimes_{(\Lambda V, d)}(\Lambda V \otimes \Lambda W, D)$, and let 
$\mu^{\prime \prime}:(\Lambda W, \bar{D}) \longrightarrow \mathcal{A}_{\mathrm{PL}}(F)$ denote the induced map. Then $\mu^{\prime \prime}$ is a quasiisomorphism, i.e., there is a commuting diagram in $\mathbf{C D G} \mathbf{A}_{\mathbb{Q}}$

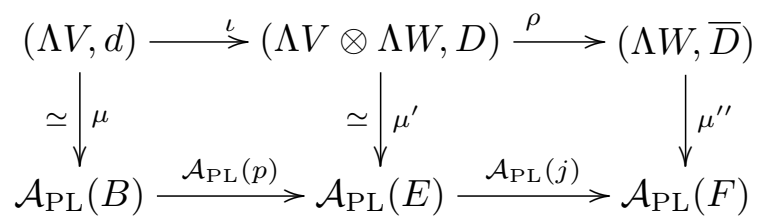

where $\rho$ is the quotient map and $j$ is the inclusion map.

(2) Given a Sullivan model $\mu:(\Lambda V, d) \longrightarrow \mathcal{A}_{\mathrm{PL}}(B)$ and a Sullivan minimal model $\mu^{\prime \prime}:(\Lambda W, d) \longrightarrow \mathcal{A}_{\mathrm{PL}}(F)$, there is a relative Sullivan algebra

$$
\iota:(\Lambda V, d) \rightarrow(\Lambda V \otimes \Lambda W, D)
$$

such that $(\Lambda W, d) \cong \mathbb{Q} \otimes_{(\Lambda V, d)}(\Lambda V \otimes \Lambda W, D)$ and a quasi-isomorphism of cochain algebras

$$
\mu^{\prime}:(\Lambda V \otimes \Lambda W, D) \longrightarrow \mathcal{A}_{\mathrm{PL}}(E)
$$

such that the diagram (2.2.1) commutes, i.e., E has a Sullivan model that is a twisted extension of a Sullivan model of the base by a Sullivan model of the fiber.

We refer the reader to Proposition 15.5 in [17] for the proof of the theorem above.

Example. Let $\Omega S^{n} \longrightarrow P S^{n} \stackrel{p}{\longrightarrow} S^{n}$ be the based path-space fibration, where $n$ is odd. Let $\mu:(\Lambda u, 0) \longrightarrow \mathcal{A}_{\mathrm{PL}}\left(S^{n}\right)$ be the Sullivan model of $S^{n}$, and consider the relative Sullivan algebra $(\Lambda u, 0) \longrightarrow(\Lambda(u, v), d)$, where $|v|=n-1$ and $d v=u$. The cochain algebra $(\Lambda(u, v), d)$ is clearly acyclic, as is $\mathcal{A}_{\mathrm{PL}}\left(P S^{n}\right)$, which implies that $\mathcal{A}_{\mathrm{PL}}(p) \circ \mu$ extends over $(\Lambda(u, v), d)$ to a quasi-isomorphism of cochain algebras $\mu^{\prime}:(\Lambda(u, v), d) \longrightarrow \mathcal{A}_{\mathrm{PL}}\left(P S^{n}\right)$. By Theorem 2.2.1, the induced cochain algebra map $\mu^{\prime \prime}:(\Lambda v, 0) \longrightarrow \mathcal{A}_{\mathrm{PL}}\left(\Omega S^{n}\right)$ is a quasi-isomorphism, which implies that $\mathrm{H}^{*}\left(\Omega S^{n} ; \mathbb{Q}\right) \cong \mathbb{Q}[v]$, when $n$ is odd.

More generally, consider the based path-space fibration $\Omega X \longrightarrow P X \stackrel{p}{\longrightarrow} X$, where $X$ is a simply connected space. Suppose that $\mu:(\Lambda V, d) \longrightarrow \mathcal{A}_{\mathrm{PL}}(X)$ is the Sullivan model of $X$.

Let $\bar{V}$ be the graded $\mathbb{Q}$-vector space, which is the suspension of $V$, i.e., $\bar{V}^{n}=$ $V^{n+1}$. Let $S$ be the derivation of $\Lambda(V \oplus \bar{V})$ specified by $S(v)=\bar{v}$ and $S(\bar{v})=0$ for all $v \in V$. Define $(\Lambda(V \oplus \bar{V}), D)$ by $D \bar{v}=-S(d v)$. Then $(\Lambda(V \oplus \bar{V}), D)$ is easily seen to be acyclic, as is $\mathcal{A}_{\mathrm{PL}}(P X)$, and therefore there is a quasi-isomorphism of cochain algebras $\mu^{\prime}:(\Lambda(V \oplus \bar{V}), D) \longrightarrow \mathcal{A}_{\mathrm{PL}}(P X)$ extending $\mathcal{A}_{\mathrm{PL}}(p) \circ \mu$. By Theorem 2.2.1, the induced map of cochain algebras $\mu^{\prime \prime}:(\Lambda \bar{V}, 0) \longrightarrow \mathcal{A}_{\mathrm{PL}}(\Omega X)$ is a quasi-isomorphism.

Theorem 2.2.1 is a consequence of the following, more general result concerning fiber squares, for which the slogan is "the model of the pullback is the pushout of the models." 
Theorem 2.2.2. Let $p: E \rightarrow B$ be a Serre fibration, where $E$ is path connected and $B$ is simply connected, with fiber $F$. Let $f: X \rightarrow B$ be a continuous map, where $X$ is simply connected. Suppose that $B$ or $F$ is of finite rational type. Consider the pullback

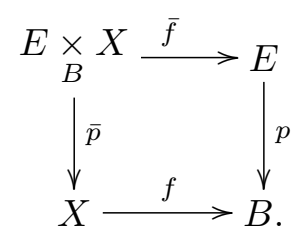

Given a commuting diagram of $C D G A$ 's

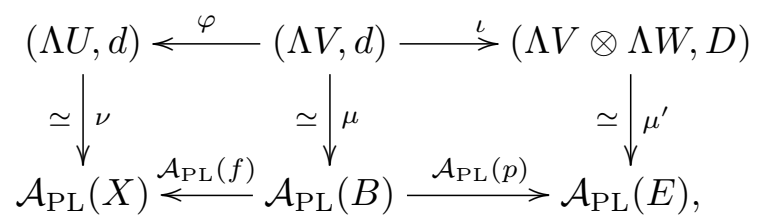

the induced map of cochain algebras

$$
(\Lambda U, d) \otimes_{(\Lambda V, d)}(\Lambda V \otimes \Lambda W, D) \longrightarrow \mathcal{A}_{\mathrm{PL}}(E \underset{B}{\times} X)
$$

is a quasi-isomorphism of CDGA's.

We again refer the reader to [17] for the proof of this theorem, in the guise of their Proposition 15.8.

Example. Let $X$ be a simply connected space of finite rational type, with Sullivan model $\varphi:(\Lambda V, d) \longrightarrow \mathcal{A}_{\mathrm{PL}}(X)$. Consider the free-loop fiber square.

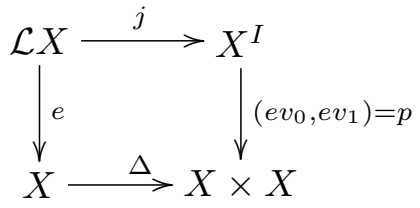

It is easy to check that

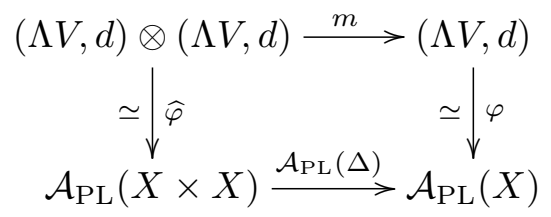

commutes, where $m$ is the multiplication map on $(\Lambda V, d)$ and $\widehat{\varphi}$ is the composite

$$
(\Lambda V, d) \otimes(\Lambda V, d) \stackrel{\varphi \otimes \varphi}{\longrightarrow} \mathcal{A}_{\mathrm{PL}}(X) \otimes \mathcal{A}_{\mathrm{PL}}(X) \stackrel{\alpha_{X}, X}{\longrightarrow} \mathcal{A}_{\mathrm{PL}}(X \times X) .
$$

Furthermore

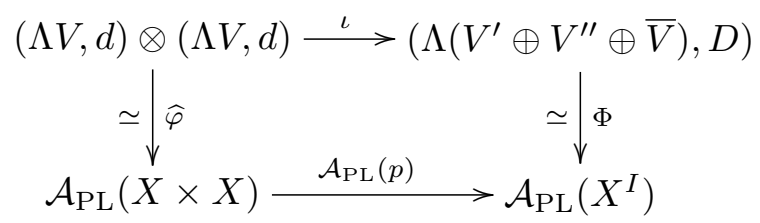


commutes as well, where $\iota$ is a relative Sullivan algebra $\left(V^{\prime}\right.$ and $V^{\prime \prime}$ are two copies of $V), \Phi$ is an appropriate extension of $\widehat{\varphi}$ and $D$ is specified as follows.

Let $S: \Lambda\left(V^{\prime} \oplus V^{\prime \prime} \oplus \bar{V}\right) \rightarrow \Lambda\left(V^{\prime} \oplus V^{\prime \prime} \oplus \bar{V}\right)$ be the derivation of degree -1 specified by $S\left(v^{\prime}\right)=\bar{v}=S\left(v^{\prime}\right)$ and $S(\bar{v})=0$. Then

$$
D(\bar{v}):=v^{\prime \prime}-v^{\prime}-\sum_{n \geq 1} \frac{(S D)^{n}}{n !}\left(v^{\prime}\right) .
$$

Applying Theorem 2.2.2 we obtain as a Sullivan model of $\mathcal{L} X$

$$
(\Lambda(V \oplus \bar{V}), \bar{D}) \cong(\Lambda V, d) \underset{(\Lambda V, d)^{\otimes 2}}{\otimes}\left(\Lambda\left(V^{\prime} \oplus V^{\prime \prime} \oplus \bar{V}\right), D\right),
$$

i.e., $\bar{D}(\bar{v})=-\bar{S}(d v)$, where $\bar{S}(v)=\bar{v}$ and $\bar{S}(\bar{v})=0$.

Sullivan and Vigué used this model to prove that if $\mathrm{H}^{*}(X ; \mathbb{Q})$ requires at least two algebra generators, then the rational Betti numbers of $\mathrm{H}^{*}(\mathcal{L} X ; \mathbb{Q})$ grow exponentially, which implies in turn that $X$ admits an infinite number of distinct closed geodesics, when $X$ is a closed Riemannian manifold [31].

\subsection{Lusternik-Schnirelmann category.}

One of the most spectacular successes of the Sullivan minimal model has been in its application to studying and exploiting the numerical homotopy invariant known as Lusternik-Schnirelmann (L.-S.) category.

Definition. A categorical covering of a space $X$ is an open cover of $X$ such that each member of the cover is contractible in $X$. The L.-S. category of a topological space $X$, denoted cat $X$, is equal to $n$ if the cardinality of the smallest categorical covering of $X$ is $n+1$.

L.-S. category is in general extremely difficult to compute. It is trivial, however, to prove that the L.-S. category of a contractible space is 0 and that cat $S^{n}=1$ for all $n$. Similarly, the L.-S. category of any suspension is 1 . More generally, a space $X$ is a co-H-space if and only if cat $X \leq 1$.

The proof of this last equivalence is most easily formulated in terms of an equivalent definition of L.-S. category, which requires the following construction, due to Ganea.

Definition. Let $p: E \rightarrow X$ be a fibration over a based topological space $\left(X, x_{0}\right)$. Let $j: F \hookrightarrow E$ denote the inclusion of the fiber of $p$ over $x_{0}$, with mapping cone $C_{j}$. Let $\hat{p}: C_{j} \rightarrow X$ denote the induced continuous map, which can be factored naturally as a homotopy equivalence followed by a fibration:

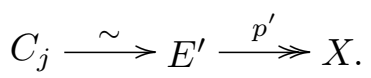

There is then a commutative diagram

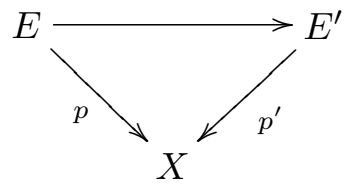


called the fiber-cofiber construction on $p$.

Let $p: P X \rightarrow X$ denote the (based) path fibration over $X$. Iterating the fibercofiber construction repeatedly leads to a commutative diagram

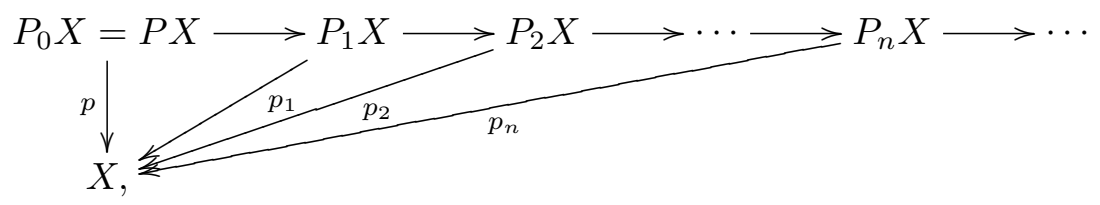

in which $P_{n} X$ is the $n^{\text {th }}$ Ganea space for $X$ and $p_{n}$ is the $n^{\text {th }}$ Ganea fibration.

Proposition 2.3.1. If $X$ is a normal space, then cat $X \leq n$ if and only if the fibration $p_{n}$ admits a section.

For a proof of this proposition, we refer the reader to Proposition 27.8 in [17].

Another equivalent definition of the L.-S. category of a based space $\left(X, x_{0}\right)$ is expressed in terms of the fat wedge on $X$

$$
T^{n} X:=\left\{\left(x_{1}, \ldots, x_{n}\right) \in X^{n} \mid \exists i \text { such that } x_{i}=x_{0}\right\} .
$$

Proposition 2.3.2. If $X$ is a path-connected $C W$-complex, then the following conditions are equivalent.

(1) cat $X \leq n$.

(2) The iterated diagonal $\Delta^{(n)}: X \longrightarrow X^{n+1}$ factors up to homotopy through the fat wedge $T^{(n+1)} X$, i.e., there is a map $\delta: X \longrightarrow T^{n+1} X$ such that the diagram

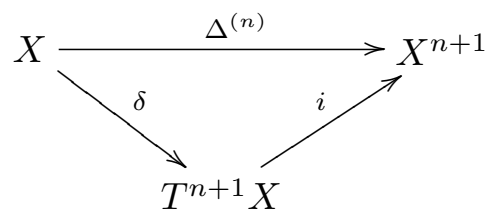

commutes up to homotopy.

We refer the reader to Proposition 27.4 in [17] for the proof of this equivalence.

Applying Proposition 2.3.2, we obtain the following useful upper bound on L.-S. category (cf., Proposition 27.5 in [17]).

Corollary 2.3.3. If $X$ is an $(r-1)$-connected $C W$-complex of dimension $d$, where $r \geq 1$, then cat $X \leq d / r$.

On the other hand, a lower bound on cat $X$ is given by the cuplength $c(X)$ of $H^{*}(X ; \mathbb{Q})$, i.e, the greatest integer $n$ such that there exist $a_{1}, \ldots, a_{n} \in H^{*}(X ; \mathbb{Q})$ satisfying $a_{1} \cup \cdots \cup a_{n} \neq 0$. We leave it as an easy exercise to prove that $c(X) \leq$ cat $X$ for all path-connected, normal spaces $X$.

Example. Observe that $c\left(\mathbb{C} P^{n}\right)=n$, so that cat $\mathbb{C} P^{n} \geq n$. On the other hand, $\mathbb{C} P^{n}$ is 1 -connected and of dimension $2 n$, implying that cat $\mathbb{C} P^{n} \leq 2 n / 2=n$. Thus, cat $\mathbb{C} P^{n}=n$.

Within the realm of rational homotopy theory, it makes sense to consider the following invariant derived from L.-S. category. 
Definition. The rational category of a simply connected space $X$, denoted cat $_{0} X$, is defined by

$$
\text { cat }_{0} X:=\min \{\operatorname{cat} Y \mid X \text { and } Y \text { have the same rational homotopy type }\} \text {. }
$$

As proved in [17] (Proposition 28.1), if $X$ is a simply connected CW-complex, then cat $_{0} X=$ cat $X_{0}$. Furthermore, it is obvious that $\operatorname{cat}_{0} X \leq$ cat $X$ for all $X$. As we show below, this inequality is sharp, i.e., there are spaces $X$ for which cat $_{0} X=$ cat $X$. On the other hand, the inequality can certainly be strict, as the case of a $\bmod p$ Moore space easily illustrates.

The next theorem has turned out to be a crucial tool in proving numerous significant results in rational homotopy theory, such as many of the dichotomy theorems (cf., Section 2.4).

Theorem 2.3.4 (The Mapping Theorem). Let $f: X \rightarrow Y$ be a continuous map between simply connected spaces. If $\pi_{*} f \otimes \mathbb{Q}$ is injective, then cat $_{0} X \leq \operatorname{cat}_{0} Y$.

The original proof of the Mapping Theorem relied on Sullivan models. There is now a purely topological and relatively simple proof, which is given in [17] (Theorem 28.6).

As a first application of the Mapping Theorem, we mention the amusing and useful corollary below, which follows immediately from the fact that the natural map from the $(n+1)^{s t}$ Postnikov fiber to the $n^{\text {th }}$ Postnikov fiber of a space induces an injection on homotopy groups.

Corollary 2.3.5. Let $X$ be a connected $C W$-complex. Let $X(n)$ denote the $n^{\text {th }}$ Postnikov fiber of $X$, for all $n \geq 1$. Then

$$
\cdots \leq \operatorname{cat}_{0} X(n+1) \leq \operatorname{cat}_{0} X(n) \leq \cdots \leq \operatorname{cat}_{0} X(2) \leq \operatorname{cat}_{0} X .
$$

One great advantage of rational category, as opposed to the usual L.-S. category, is that it can explicitly calculated in terms of the Sullivan model, as stated in the next theorem.

Theorem 2.3.5. Let $\varphi:(\Lambda V, d) \stackrel{\sim}{\longrightarrow} \mathcal{A}_{\mathrm{PL}}(X)$ be the Sullivan minimal model of a simply connected space $X$ of finite rational type. Let $\left(\Lambda V / \Lambda^{>n} V, \bar{d}\right)$ denote the $C D G A$ obtained by taking the quotient of $(\Lambda V, d)$ by the ideal of words of length greater than $n$, and let

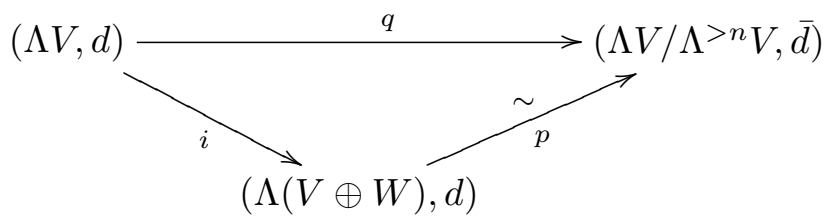

be a factorization of the quotient map $q$ as a relative Sullivan algebra, followed by a surjective quasi-isomorphism. Then cat $_{0} X \leq n$ if and only if $i$ admits a CDGA retraction $\rho:(\Lambda(V \oplus W), d) \longrightarrow(\Lambda V, d)$, i.e., $\rho i=I d_{(\Lambda V, d)}$.

The fat wedge formulation of the definition of L.-S. category is crucial in the proof of this theorem, for which we refer the reader to Propositions 29.3 and 29.4 in $[17]$. 
Examples. Since $H^{n}\left(S^{n} ; \mathbb{Q}\right)=\mathbb{Q}$, the rationalization $S_{0}^{n}$ of the $n$-sphere is not contractible and therefore cat $S^{n}=$ cat $S_{0}^{n}>0$. On the other hand, cat $_{0} S^{n} \leq$ cat $S^{n}=1$, whence cat $_{0} S^{n}=1$, providing the promised example of equality between rational category and L.-S. category of a space.

This calculation can also be carried out easily using the Sullivan model (cf., Section 2.1). If $n$ is odd, then the Sullivan model is $(\Lambda(x), 0)$, where $x$ is of degree $n$. Observe that $\Lambda(x) / \Lambda^{>1}(x)$ is isomorphic to $\Lambda(x)$, since $x$ is of odd degree. Since the quotient map $q$ is itself the identity map in this case, it follows trivially that $\operatorname{cat}_{0} S^{n} \leq 1$.

If $n$ is even, the relevant Sullivan model is $(\Lambda(y, z), d)$, where $\operatorname{deg} y=n, \operatorname{deg} z=$ $2 n-1$ and $d z=y^{2}$. An easy calculation shows that

$$
\left(\Lambda(y, z) / \Lambda^{>1}(y, z), \bar{d}\right)=(\mathbb{Q} \oplus \mathbb{Q} \cdot y \oplus \mathbb{Q} \cdot z, 0) .
$$

It is not too difficult to show that the quotient map $q$ factors as

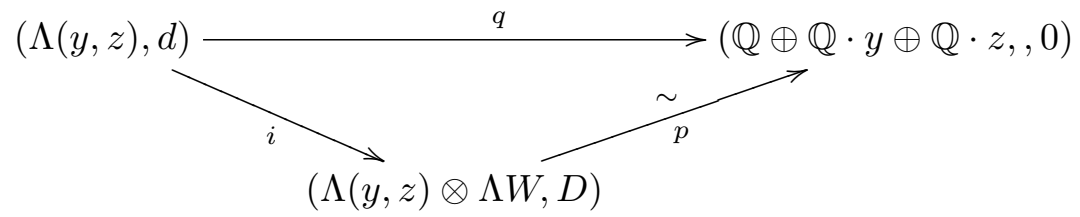

where $D W \subset \Lambda(y, z) \otimes \Lambda^{+} W$, i.e., the differential of any generator of $W$, if nonzero, is a sum of words, all of which contain at least one letter from $W$. We can therefore define a CDGA retraction $\rho$ by setting $\rho(w)=0$ for all $w \in W$, implying that cat $_{0} S^{n} \leq 1$.

Though Theorem 2.3.5 does simplify the calculation of rational category by making it purely algebraic, the computations involved are still difficult, which led Halperin and Lemaire to propose the following, apparently weaker numerical invariant of rational homotopy [22].

Definition. Let $X$ be a simply connected space of finite rational type, with Sullivan model $\varphi:(\Lambda V, d) \stackrel{\sim}{\longrightarrow} \mathcal{A}_{\mathrm{PL}}(X)$. If the map $i$ in diagram (2.3.1) admits a retraction as morphisms in the category of $(\Lambda V, d)$-modules, then Mcat $_{0} X \leq n$.

As it turned out, however, the apparent weakness of Mcat ${ }_{0}$ was only an illusion.

Theorem 2.3.6. Mcat $_{0} X=$ cat $_{0} X$ for all simply connected spaces $X$ of finite rational type.

For the proof of this theorem, which requires a deep understanding of the factorization of the quotient map, we refer the reader to Theorem 29.9 in [17].

Theorem 2.3.6 implies that to show that cat $_{0} X \leq n$, it suffices to find a $(\Lambda V, d)$ module retraction of $i$ in diagram (2.3.1), which has proven to be a very effective simplification. We next outline briefly one application of this simplification, to the study of the additivity of L.-S. category.

It is not difficult to show that $\operatorname{cat}(X \times Y) \leq$ cat $X+$ cat $Y$ for all normal spaces $X$ and $Y$. At the end of the 1960's Ganea observed that in the only known examples for which $\operatorname{cat}(X \times Y) \neq$ cat $X+\operatorname{cat} Y$, the spaces $X$ and $Y$ had homology torsion at distinct primes. He conjectured therefore that $\operatorname{cat}\left(X \times S^{n}\right)=\operatorname{cat} X+1$ for all spaces $X$ and all $n \geq 1$, since $S^{n}$ has no homology torsion whatsoever.

In fact, as stated precisely below, if we forget torsion completely and work rationally, then L.-S. category is indeed additive. 
Theorem 2.3.7. If $X$ and $Y$ are simply connected topological spaces of finite rational type, then $\operatorname{cat}_{0}(X \times Y)=\operatorname{cat}_{0} X+\operatorname{cat}_{0} Y$.

The proof of this theorem depends in an essential way on Theorem 2.3.6. We refer the reader to Sections 29(h) and 30(a) in [17] for further details.

As an epilogue to this story of Sullivan minimal models and L.-S. category, we mention that in 1997 Iwase applied classical homotopy-theoretic methods to the construction of a counter-example to Ganea's conjecture [14]. In particular, he built a 2-cell complex $X$ such that $\operatorname{cat}\left(X \times S^{n}\right)=2=$ cat $X$.

\subsection{Dichotomy.}

There is a beautiful dichotomy governing finite $\mathrm{CW}$-complexes in rational homotopy theory, expressed as follows: the rational homotopy groups of a finite CWcomplex either are of finite total dimension as graded rational vector space or grow exponentially. We first examine the former case, that of elliptic spaces, then the latter case, that of hyperbolic spaces.

Definition. A simply connected topological space $X$ is rationally elliptic if

$$
\operatorname{dim} \mathrm{H}^{*}(X ; \mathbb{Q})<\infty \text { and } \operatorname{dim} \pi_{*}(X) \otimes \mathbb{Q}<\infty .
$$

The formal dimension fdim $X$ of a rationally elliptic space $X$ is defined by

$$
\operatorname{fdim} X:=\max \left\{k \mid \mathrm{H}^{k}(\Lambda V, d) \neq 0\right\} .
$$

The even exponents of a rationally elliptic space $X$ are the postive integers $a_{1}, \ldots, a_{q}$ such that there is a basis $\left(y_{j}\right)_{1 \leq j \leq q}$ of $\pi_{\text {even }} X \otimes \mathbb{Q}$ with $\operatorname{deg} y_{j}=2 a_{j}$. Similarly, the odd exponents of $X$ are the positive integers $b_{1}, \ldots, b_{p}$ such that there is a basis $\left(x_{i}\right)_{1 \leq i \leq p}$ of $\pi_{\text {odd }} X \otimes \mathbb{Q}$ with $\operatorname{deg} x_{i}=2 b_{i}-1$.

Examples. Spheres, complex projective spaces, products of elliptic spaces, and homogeneous spaces are examples of elliptic spaces.

The following special case of elliptic spaces is important for understanding general elliptic spaces.

Definition. A CDGA $(\Lambda V, d)$ is pure if $\operatorname{dim} V<\infty,\left.d\right|_{V^{\text {even }}}=0$ and $d\left(V^{\text {odd }}\right) \subseteq$ $\Lambda V^{\text {even }}$. A space is pure if its Sullivan model is pure.

A pure CDGA $(\Lambda V, d)$ admits a differential filtration $F_{k}(\Lambda V, d)=\Lambda V^{\text {even }} \otimes$ $\Lambda \leq k V^{\text {odd }}$. In particular, $d\left(\Lambda V^{\text {even }} \otimes \Lambda^{k} V^{\text {odd }}\right) \subseteq \Lambda V^{\text {even }} \otimes \Lambda^{k-1} V^{\text {odd }}$. Write

$$
\mathrm{H}_{k}(\Lambda V, d)=\frac{\operatorname{ker}\left(d: \Lambda V^{\text {even }} \otimes \Lambda^{k} V^{\text {odd }} \rightarrow \Lambda V^{\text {even }} \otimes \Lambda^{k-1} V^{\text {odd }}\right)}{\operatorname{Im}\left(d: \Lambda V^{\text {even }} \otimes \Lambda^{k+1} V^{\text {odd }} \rightarrow \Lambda V^{\text {even }} \otimes \Lambda^{k} V^{\text {odd }}\right)} .
$$

The following list of the most important properties of pure CDGA's summarizes Propositions 32.1 and 32.2 in [17].

Proposition 2.4.1. Let $(\Lambda V, d)$ be a pure $C D G A$.

(1) $\operatorname{dim} \mathrm{H}^{*}(\Lambda V, d)<\infty \Leftrightarrow \operatorname{dim} H_{0}(\Lambda V, d)<\infty$.

(2) If $\operatorname{dim} \mathrm{H}^{*}(\Lambda V, d)<\infty$, then $\mathrm{H}^{n}(\Lambda V, d)$ is a 1-dimensional subspace of $\mathrm{H}_{r}(\Lambda V, d)$, where $n$ is the formal dimension of $(\Lambda V, d)$ and $r=\max \left\{k \mid \mathrm{H}_{k}(\Lambda V, d) \neq 0\right\}$.

(3) $r=\operatorname{dim} V^{\text {odd }}-\operatorname{dim} V^{\text {even }}$, thus

$$
\operatorname{dim} V^{\text {odd }}=\operatorname{dim} V^{\text {even }} \Leftrightarrow \mathrm{H}^{*}(\Lambda V, d)=\mathrm{H}_{0}(\Lambda V, d) .
$$

(4) $\operatorname{fdim} X=\sum_{i}\left(2 b_{i}-1\right)-\sum_{j}\left(2 a_{j}-1\right)$. 
We present next a tool for determining whether spaces are elliptic, based on the notion of pure spaces.

Definition. Let $(\Lambda V, d)$ be a Sullivan algebra such that $\operatorname{dim} V<\infty$. Filter $(\Lambda V, d)$ by

$$
\mathcal{F}^{p}(\Lambda V, d)=\bigoplus_{k+l \geq p}\left(\Lambda V^{\text {even }} \otimes \Lambda^{k} V^{\text {odd }}\right)^{l} .
$$

The induced spectral sequence is the odd spectral sequence and converges to $\mathrm{H}^{*}(\Lambda V, d)$.

Observe that the $E_{0}$-term of the odd spectral sequence is the associated graded of $\left(\Lambda V, d_{\sigma}\right)$, where $d_{\sigma}\left(V^{\text {even }}\right)=0, d_{\sigma}\left(V^{\text {odd }}\right) \subseteq \Lambda V^{\text {even }}$ and $\left(d-d_{\sigma}\right)\left(V^{\text {odd }}\right) \subseteq \Lambda V^{\text {even }} \otimes$ $\Lambda^{+} V^{\text {odd }}$. We call $\left(\Lambda V, d_{\sigma}\right)$ the associated pure Sullivan algebra of $(\Lambda V, d)$.

Proposition 2.4.2. Under the hypotheses of the definition above,

$$
\operatorname{dim} \mathrm{H}^{*}(\Lambda V, d)<\infty \Leftrightarrow \operatorname{dim} \mathrm{H}^{*}\left(\Lambda V, d_{\sigma}\right)<\infty .
$$

Thus, $(\Lambda V, d)$ is elliptic if and only if $\left(\Lambda V, d_{\sigma}\right)$ is elliptic.

Proof. Since the odd spectral sequence converges from $\mathrm{H}^{*}\left(\Lambda V, d_{\sigma}\right)$ to $\mathrm{H}^{*}(\Lambda V, d)$, one implication is clear. An algebraic version of the Mapping Theorem (Theorem 2.3.4) plays an essential role in the rest of the proof. We refer the reader to Proposition 32.4 in [17] for the complete proof.

Example. Consider $(\Lambda V, d)=\left(\Lambda\left(a_{2}, x_{3}, u_{3}, b_{4}, v_{5}, w_{7}\right), d\right)$, where the subscript of a generator equals its degree and $d$ is specified by $d a=0, d x=0, d u=a^{2}$, $d b=a x, d v=a b-u x$ and $d w=b^{2}-w x$. Its associated pure Sullivan algebra is $\left(\Lambda(a, x, u, b, v, w), d_{\sigma}\right)$, where $d_{\sigma} a=0, d_{\sigma} x=0, d_{\sigma} u=a^{2}, d_{\sigma} b=0, d_{\sigma} v=a b$ and $d_{\sigma} w=b^{2}$. A straightforward calculation shows that

$$
\mathrm{H}^{*}\left(\Lambda V, d_{\sigma}\right)=\mathbb{Q} \cdot a \oplus \mathbb{Q} \cdot b \oplus \Lambda y /\left(y^{3}\right) \oplus \Lambda z /\left(z^{3}\right),
$$

where $y$ is represented by $b u-a v$ and $z$ is represented by $a w-b v$. In particular $\operatorname{dim} \mathrm{H}^{*}\left(\Lambda V, d_{\sigma}\right)<\infty$, which implies that $(\Lambda V, d)$ is elliptic.

The next theorem describes the amazing numerology of elliptic spaces, which imposes formidable constraints on their form.

Theorem 2.4.3. Let $(\Lambda V, d)$ be an elliptic Sullivan algebra of formal dimension $n$ and with even and odd exponents $a_{1}, \ldots, a_{q} ; b_{1}, \ldots, b_{p}$. Then:

(1) $\sum_{i=1}^{p}\left(2 b_{i}-1\right)-\sum_{j=1}^{q}\left(2 a_{j}-1\right)=n$;

(2) $\sum_{j=1}^{q} 2 a_{j} \leq n$;

(3) $\sum_{i=1}^{p}\left(2 b_{1}-1\right) \leq 2 n-1$; and

(4) $\operatorname{dim} V^{\text {even }} \leq \operatorname{dim} V^{\text {odd }}$.

As a consequence of this theorem, we know, for example, that if $(\Lambda V, d)$ is an elliptic Sullivan algebra of formal dimension $n$, then $V=V^{\leq 2 n-1}, \operatorname{dim} V^{>n} \leq 1$ and $\operatorname{dim} V \leq n$.

Remarks on the proof. One first proves by induction on $\operatorname{dim} V$ that the formal dimensions of $(\Lambda V, d)$ and of its associated pure tower are the same, reducing the proof of the theorem to the pure case. For further details, we refer the reader to Theorem 32.6 in [17]. 
Definition. The Euler-Poincaré characteristic of a graded vector space $W$ is the integer

$$
\chi_{W}=\sum_{i}(-1) \operatorname{dim} W^{i}=\operatorname{dim} W^{\text {even }}-\operatorname{dim} W^{\text {odd }} .
$$

It is easy to show that $\chi_{W}=\chi_{\mathrm{H}^{*}(W, d)}$, for any choice of differential $d$ on $W$.

Proposition 2.4.4. If $(\Lambda V, d)$ is an elliptic Sullivan algebra, then $\chi_{\Lambda V} \geq 0$ and $\chi_{V} \leq 0$. Furthermore, the following statements are equivalent.

(1) $\chi_{\Lambda V}>0$.

(2) $\mathrm{H}^{*}(\Lambda V, d)=\mathrm{H}^{\text {even }}(\Lambda V, d)$.

(3) $\mathrm{H}^{*}(\Lambda V, d)=\Lambda\left(y_{1}, \ldots, y_{q}\right) /\left(u_{1}, \ldots, u_{p}\right)$, where $\left(u_{1}, \ldots, u_{p}\right)$ is a regular sequence.

(4) $(\Lambda V, d)$ is isomorphic to a pure complex.

(5) $\chi_{V}=0$.

Remarks on the proof. The proof of this proposition reduces essentially to Poincaré series calculations. We refer the reader to Proposition 32.10 in [17] for details of the calculations.

Application to free torus actions. (Example 3 in section 32(e) of [17]) Let $T$ denote the $r$-torus, i.e., the product of $r$ copies of $S^{1}$. Suppose that $T$ acts smoothly and freely on a simply connected, compact, smooth manifold $M$. There exists then a smooth principal bundle $M \rightarrow M / T$ and thus a classifying map $M / T \rightarrow B T$ with homotopy fiber $M$.

If $M$ is elliptic, then $M / T$ is also elliptic, since $M / T$ is compact and $B T=$ $\left(\mathbb{C} P^{\infty}\right)^{r}$. Furthermore,

$$
0 \geq \chi_{\pi}(M / T)=\chi_{\pi}(M)+\chi_{\pi}(B T)=\chi_{\pi}(M)+r
$$

implying that $r \leq-\chi_{\pi}(M)$.

Now we go to the other extreme.

Definition. A simply connected space $X$ with the homotopy type of a finite CWcomplex is rationally hyperbolic if $\operatorname{dim} \pi_{*}(X) \otimes \mathbb{Q}=\infty$.

The following theorem, which justifies the terminology "hyperbolic," is Theorem 33.2 in [17]. Its proof depends strongly on the Mapping Theorem (Theorem 2.3.4).

Theorem 2.4.5. If $X$ is a rationally hyperbolic space, then there exist $C>1$ and $N \in \mathbb{Z}$ such that

$$
\sum_{i=0}^{n} \operatorname{dim} \pi_{i}(X) \otimes \mathbb{Q} \geq C^{n}
$$

for all $n \geq N$.

In other words, the rational homotopy groups of $X$ grow exponentially. Moreover, as stated more precisely in the next theorem (Theorem 33.3 in [17]), there are no "long gaps" in the rational homotopy groups of $X$. 
Theorem 2.4.6. If $X$ is a rationally hyperbolic space of formal dimension $n$, then for all $k \geq 1$, there exists $i \in(k, k+n)$ such that $\pi_{i} X \otimes \mathbb{Q} \neq 0$. Furthermore, for $k \gg 0$,

$$
\sum_{i=k+1}^{k+n-1} \operatorname{dim} \pi_{i}(X) \otimes \mathbb{Q} \geq \frac{\operatorname{dim} \pi_{k} X \otimes \mathbb{Q}}{\operatorname{dim} \mathrm{H}^{*}(X ; \mathbb{Q})} .
$$

Consequently, if $X$ has formal dimension $n$, then

$$
X \text { is rationally elliptic } \Leftrightarrow \pi_{j}(X) \otimes \mathbb{Q}=0 \quad \forall j \in[2 n, 3 n-2],
$$

a simple and lovely test of ellipticity.

\section{Commutative algebra And Rational homotopy theory}

In the late 1970's two algebraists, Luchezar Avramov of the University of Sofia and Jan-Erik Roos of the University of Stockholm, discovered and began to exploit a deep connection between local algebra and rational homotopy theory. In 1981 they established contact with the rational homotopy theorists, initiating a powerful synergy that led to a multitude of important results in both fields. In this section, of a more expository nature than the preceding sections, we describe certain of the most important results of this collaboration. For further details we refer the reader to Section 4 of [23].

Roos' interest in rational homotopy theory was inspired by Jean-Michel Lemaire's work on Serre's question concerning Poincaré series of the rational homology of loop spaces and by the work of local algebraists on the analogous question of Kaplansky and Serre for local rings. More precisely, Lemaire had studied the Poincaré series

$$
\sum_{n \geq 0} \operatorname{dim}_{\mathbb{Q}} H_{n}(\Omega E ; \mathbb{Q}) \cdot z^{n}
$$

for $E$ a finite, simply connected $\mathrm{CW}$-complex, while local algebraists were interested in the series

$$
\sum_{n \geq 0} \operatorname{dim}_{\mathbb{k}} \operatorname{Ext}_{R}^{n}(\mathbb{k}, \mathbb{k}) \cdot z^{n}
$$

for $R$ a local, commutative, noetherian ring with residue field $\mathbb{k}$. In both cases, the goal was to determine under what conditions the series represented a rational function.

Roos established a research program to study the homological properties of local rings, in particular those whose maximal ideal $\mathfrak{m}$ satisfied $\mathfrak{m}^{3}=0$, the first nontrivial case for Poincaré series calculations. He realized that in order to study local rings, it was useful, or even necessary, to work in the larger category of (co)chain algebras. By 1976 he had proved the equivalence of Serre's problem for CW-complexes $E$ such that $\operatorname{dim} E=4$ and of the Kaplansky-Serre problem for local rings $(R, \mathfrak{m})$ such that $\mathfrak{m}^{3}=0[29]$.

Avaramov ascribes his original interest in rational homotopy theory to Levin's result from 1965 that if $R$ is a local, commutative ring with residue field $\mathbb{k}$, then $\operatorname{Tor}^{R}(\mathbb{k}, \mathbb{k})$ is a graded, divided powers Hopf algebra [26]. Since the dual of a graded, divided powers Hopf algebra is the universal enveloping algebra of a uniquely defined graded Lie algebra ( char $\mathbb{k}=0[28]$, char $\mathbb{k}>2[7]$, char $\mathbb{k}=2[30]$ ), it is possible 
to associate to any noetherian, local, commmutative ring $R$ a uniquely defined graded Lie algebra $\pi^{*}(R)$, the homotopy Lie algebra of $R$. Based on results in characteristic 0 due to Gulliksen in the late 1960's, Avramov proved that if $R \rightarrow S$ is a homomorphism of noetherian, local, commutative rings with the same residue field $\mathbb{k}$ such that $S$ is $R$-flat, then there is an exact sequence of groups

$$
\ldots \rightarrow \pi^{n}\left(S \otimes_{R} \mathbb{k}\right) \rightarrow \pi^{n}(S) \rightarrow \pi^{n}(R) \stackrel{\delta_{n}}{\longrightarrow} \pi^{n+1}\left(S \otimes_{R} \mathbb{k}\right) \rightarrow \ldots
$$

The existence of such a long exact sequence of homotopy groups confirmed Avramov's intuition that rational homotopy invariants provided the correct analogy for homology invariants of local rings in arbitrary characteristic.

In 1980 David Anick, constructed a finite, simply connected CW-complex $E$ of dimension $\leq 4$ such that the Poincaré series of the homology of $\Omega E$ was not rational, thus answering Serre's question [2]. Anick's construction interested rational homotopy theorists because of its relation to the dichotomy between elliptic and hyperbolic spaces; see Section 2.4. Local algebraists were interested because of Roos's result, which allowed the transcription of Anick's space into a local ring $(R, \mathfrak{m})$ with $\mathfrak{m}^{3}=0$ and with irrational Poincaré series. Shortly after Anick's result became known, Roos and his student Clas Löfwall discovered other examples of local rings with irrational Poincaré series that they obtained by completely different methods [27].

The converging interests of rational homotopy theorists and of local algebraists led to direct contact between the two groups in 1981. Inspired by the work of Roos and his colleagues, Félix and Thomas began to work on calculating the radius of convergence of the Poincaré series of a loop space [18], establishing the following beautiful characterization, the proof of which relies heavily on results from []: a simply connected space $E$ is rationally elliptic if and only if the radius of convergence of the Poincaré series of $\Omega E$ is 1 . If $E$ is rationally hyperbolic, then the radius of convergence is strictly less than 1 . Moreover, they found a relatively easily computable upper bound for the radius of convergence in the case of a hyperbolic, formal space. They also showed that if $A$ is a noetherian, connected graded commutative algebra over a field $\mathbb{k}$ of characteristic zero and $\rho_{A}$ denotes the radius of convergence of

$$
P_{A}(z)=\sum_{n \geq o} \operatorname{dim} \operatorname{Tor}_{n}^{A}(\mathbb{k}, \mathbb{k}) \cdot z^{n}
$$

then either $\rho_{A}=+\infty$ and $A$ is a polynomial algebra; or $\rho_{A}=1, A$ is a complete intersection, and the coefficients of $P_{A}(z)$ grow polynomially; or $\rho_{A}<1, A$ is not a complete intersection, and the coefficients of $P_{A}(z)$ grow exponentially. Avramov later generalized this result to any characteristic.

The written version of Avramov's Luminy talk on the close links between local algebra and rational homotopy theory provides an excellent and thorough introduction to the subject [5]. His article contains the first "dictionary" between rational homotopy theory and local algebra, explaining how to translate notions and techniques from one field to the other. Given a theorem in one field, applying the dictionary leads to a statement in the other field that stands a reasonable chance of being true, though the method of proof may be completely different.

Avramov and Halperin wrote another thorough introduction to the subject in the proceedings of the Stockholm conference of 1983[7]. It begins at a more elementary level than the survey article of Avramov in the proceedings of the Luminy 
conference, leading the reader from first principles of differential graded homological algebra to notions of homotopy fiber and loop space and on to the homotopy Lie algebra.

In his introductory article [5] Avramov emphasized the importance of minimal models in local ring theory. If $K^{R}$ is the Koszul complex of a local, commutative ring such that the Yoneda algebra $\operatorname{Ext}_{R}^{*}(\mathbb{k}, \mathbb{k})$ is noetherian, then there is a minimal, commutative cochain algebra $(\Lambda V, d)$ over the residue field of $R$ that is quasi-isomorphic to $K^{R}$. Avaramov called $(\Lambda V, d)$ the minimal model of $R$. He established its relevance by observing that in degrees greater than 1 , the graded Lie algebra derived from $\left(\Lambda V, d_{2}\right)$ was isomorphic to the homotopy Lie algebra of $R$.

In [16] Félix, Halperin, and Thomas continued the in-depth study of the homotopy Lie algebra of a rationally hyperbolic space begun by Félix and Halperin in [13]. They showed, for example, that if $E$ is rationally hyperbolic, then its rational homotopy Lie algebra is not solvable. Moreover they proposed as conjectures translations of their theorems into local algebra, where, for a local ring $(R, \mathfrak{m})$ with residue field $\mathbb{k}$, L.-S. category is replaced by $\operatorname{dim}_{\mathbb{k}}\left(\mathfrak{m} / \mathfrak{m}^{2}\right)-\operatorname{depth} R$ and infinite dimensional rational homotopy is replaced by $R$ not being a complete intersection. Recall that

$$
\operatorname{depth} R=\inf \left\{j \mid \operatorname{Ext}_{R}^{j}(\mathbb{k}, R) \neq 0\right\} .
$$

Avramov and Halperin quickly proved a weaker version of one of the conjectures in [16], when they showed that $R$ is a complete intersection if and only if its homotopy Lie algebra is nilpotent $[6]$.

In [16] Félix, Halperin and Thomas also mentioned a very important conjecture due to Avramov and Félix, stating that the homotopy Lie algebra of a rationally hyperbolic space should contain a free Lie algebra on at least two generators. This conjecture has motivated much interesting work in the study of the homotopy Lie algebra and has not as yet (2005) been proved.

Using minimal model techniques, Halperin and Bøgvad proved two conjectures due to Roos, which are "translations" of each other [8]. They showed that

(1) if $R$ is a local, commutative ring such that the Yoneda algebra $\operatorname{Ext}_{R}^{*}(\mathbb{k}, \mathbb{k})$ is noetherian, then $R$ is a complete intersection; and

(2) if $E$ is a simply connected, finite $\mathrm{CW}$-complex such that the Pontryagin algebra $H_{*}(\Omega E ; \mathbb{Q})$ is noetherian, then $E$ is rationally elliptic.

Their proof is based on a slightly weakened form of the Mapping Theorem that holds over a field of any characteristic, as well as on ideas from the article of Félix, Halperin and Thomas of the previous year [16].

In the spring of 1985 Halperin applied minimal model techniques to answering on old question concerning the deviations of a local ring [21]. The $j$ th deviation, $e_{j}$, of a noetherian, local, commutative ring $R$ with residue field $\mathbb{k}$ is $\operatorname{dim}_{\mathbb{k}} \pi^{j}(R)$. Assmus had shown in 1959 that $R$ is a weak complete intersection if and only if $e_{j}=0$ for all $j>2$ [3], raising the question of whether any deviation could vanish if $R$ were not a weak complete intersection. Halperin succeeded in answering this question, showing that if $R$ is not a weak complete intersection, then $e_{j} \neq 0$ for all $j$.

The Five Author paper [14] represents a great leap forward in understanding of the structure of the homotopy Lie algebra of a space or of a local ring. The principal innovation of the Five Author paper consists in exploiting the radical of 
the homotopy Lie algebra, i.e., the sum of all of its solvable ideals, which rational homotopy theorists had begun to study in 1983. The radical itself is in general not solvable.

Expressed in topological terms, the main theorem of the Five Author paper states that if $E$ is a simply connected $C W$-complex of finite type and cat $(E)=m<\infty$, then the radical of the homotopy Lie algebra, $\operatorname{Rad}(E)$ is finite dimensional and $\operatorname{dim} \operatorname{Rad}(E)_{\text {even }} \leq m$. This is a consequence of two further theorems, both of which are of great interest themselves. The first concerns the relations among the rational L.-S. category of a space and the depth and global dimension of its homotopy Lie algebra. Recall that the gobal dimension of a local $\operatorname{ring} R$ with residue field $\mathbb{k}$ is defined by

$$
\text { gl. } \operatorname{dim} .(R)=\sup \left\{j \mid \operatorname{Ext}_{R}^{j}(\mathbb{k}, \mathbb{k}) \neq 0\right\} .
$$

The precise statement of this theorem in topological terms is then that if $L$ is the homotopy Lie algebra of a simply-connected CW-complex of finite type $E$, then either

$$
\operatorname{depth} U L<\operatorname{cat}_{0}(E)<\text { gl. } \operatorname{dim} . U L
$$

or

$$
\operatorname{depth} U L=\operatorname{cat}_{0}(E)=\text { gl. } \operatorname{dim} . U L .
$$

The second theorem states that under the same hypotheses, if depth $U L<\infty$, then $\operatorname{Rad}(E)$ is finite dimensional and satisfies $\operatorname{dim} \operatorname{Rad}(E)_{\text {even }} \leq \operatorname{depth} U L$. Moreover, if $\operatorname{dim} \operatorname{Rad}(E)_{\text {even }}=\operatorname{depth} U L$, then $\operatorname{Rad}(E)=L$.

Rational homotopy theorists have exploited extensively the results of [14] in developing a deep understanding of the homotopy Lie algebra of rationally hyperbolic spaces. The methods the five authors devised to prove their results have turned out to be extremely important as well. For example, since their goal was to relate $\operatorname{cat}_{0}(E)$ to $\operatorname{depth}(L)$, they needed to construct a model of the quotient cochain algebra $\left(\Lambda V / \Lambda^{>n} V, \bar{d}\right)$, where $(\Lambda V, d)$ is the Sullivan minimal model of $E$. Their method for doing so, based on perturbation of a model for $\left(\Lambda V / \Lambda^{>n} V, \overline{d_{2}}\right)$, proved to be useful in a number of other circumstances, such as in the proof of Theorem 2.3.6.

\section{REFERENCES}

[1] M. André, Cohomologie des algèbres différentielles où opère une algèbre de Lie, Tôhoku Math. J. 14 (1962), 263-311.

[2] D. J. Anick, A counter-example to a conjecture of Serre, Annals of Math. 115 (1982), 1-33.

[3] E. Assmus, On the homology of local rings, Ill. J. Math. 3 (1959), 187-199.

[4] L. Avramov, Homology of local flat extensions and complete intersection defects, Math. Ann. 228 (1977), 27-37.

[5] L. Avramov, Local algebra and rational homotopy, Homotopie Algébrique et Algèbre Locale, Astérisque, vol. 113-114, 1984, pp. 16-43.

[6] L. Avramov and S. Halperin, On the structure of the homotopy Lie algebra of a local ring, Homotopie Algébrique et Algèbre Locale, Astérisque, vol. 113-114, 1984, pp. 153-155.

[7] L. Avramov and S. Halperin, Through the looking glass: a dictionary between rational homotopy theory and local algebra, Algebra, Algebraic Topology and their Interactions, Springer Lecture Notes in Mathematics, vol. 1183, 1986, pp. 1-27.

[8] R. Bøgvad and S. Halperin, On a conjecture of Roos, Algebra, Algebraic Topology and their Interactions, Springer Lecture Notes in Mathematics, vol. 1183, 1986, pp. 120-126.

[9] A. K. Bousfield and V. K. A. M. Gugenheim, On PL De Rham Theory and Rational Homotopy Type, Memoirs of the A. M. S., vol. 179, 1976. 
[10] E. Cartan, Sur les nombres de Betti des espaces de groupes close, C.R. Acad. Sci. Paris 187 (1928), 196-197.

[11] P. Deligne, P. Griffiths, J. Morgan and D. Sullivan, Real homotopy theory of Kähler manifolds, Inventiones 29 (1975), 245-274.

[12] W. Dwyer and J. Spalinski, Homotopy theories and model categories, Handbook of Algebraic Topology (I. M. James, ed.), North-Holland, 1995, pp. 73-126.

[13] Y. Félix and S. Halperin, Rational L.-S. category and its applications, Trans. A.M. S. 273 (1982), 1-37.

[14] Y. Félix, S. Halperin, C. Jacobsson, C. Löfwall, and J.-C. Thomas, The radical of the homotopy Lie algebra, Amer. J. Math. 110 (1988), 301-322.

[15] Y. Félix, S. Halperin and J.-M. Lemaire, Rational category and conelength of Poincaré complexes, Topology 37 (1998), 743-748.

[16] Y. Félix, S. Halperin and J.-C. Thomas, The homotopy Lie algebra for finite complexes, Publ. Math. I. H. E. S. 56 (1982), 387-410.

[17] Y. Félix, S. Halperin and J.-C. Thomas, Rational Homotopy Theory, Graduate Texts in Mathematics, vol. 205, Springer-Verlag, 2001.

[18] Y. Félix and J.-C. Thomas, The radius of convergence of Poincaré series of loop spaces, Invent. Math. 68 (1982), 257-274.

[19] P. Goerss, Model categories and simplicial methods, Contemporary Math. (2006).

[20] V.K.A.M Gugenheim and H. J. Munkholm, On the extended functoriality of Tor and Cotor, J. Pure Appl. Algebra 4 (1974), 9-29.

[21] S. Halperin, The nonvanishing of deviations of a local ring, Comment. Math. Helv. 62 (1987), 646-653.

[22] S. Halperin and J.-M. Lemaire, Notions of category in differential algebra, Algebraic Topology: Rational Homotopy, Springer Lecture Notes in Mathematics, vol. 1318, pp. 138-153.

[23] K. Hess, A history of rational homotopy theory, History of Topology, Elsevier Science B.V., 1999, pp. $757-796$.

[24] M. Hovey, Model Categories, Mathematical Surveys and Monographs, vol. 63, American Mathematical Society, 1999.

[25] N. Iwase, Ganea's conjecture on Lusternik-Schnirelmann category, Bull. London Math. Soc. 30 (1998), 623-634.

[26] G. Levin, Homology of local rings, Ph. D. Thesis, Univ. Chicago, 1965.

[27] C. Löfwall and J.-E. Roos, Cohomologie des algèbres de Lie graduées et séries de PoincaréBetti non rationnelles, C. R. Acad. Sci. Paris 290 (1980), 733-736.

[28] J. Milnor and J. Moore, On the structure of Hopf algebras, Annals of Math. 81 (1965), 211-264.

[29] J.-E. Roos, Relations between the Poincaré-Betti series of loop spaces and local rings, Séminaire d'algèbre Paul Dubreil, Springer Lecture Notes in Mathematics, vol. 740, pp. 285-322.

[30] G. Sjödin, Hopf algebras and derivations, J. Algebra 64 (1980), 218-229.

[31] D. Sullivan and M. Vigué-Poirrier, The homology theory of the closed geodesic problem, J. Differential Geometry 11 (1976), 633-644. 